\title{
GiovanNi PROUSE
}

\section{Sur l'écoulement d'un liquide visqueux incompressible dans un tuyau avec paroi perméable et déformable}

Mémoires de la S. M. F., tome 31-32 (1972), p. 317-319

<http://www.numdam.org/item?id=MSMF_1972_31-32_317_0>

(C) Mémoires de la S. M. F., 1972, tous droits réservés.

L'accès aux archives de la revue « Mémoires de la S. M. F. » (http://smf. emath.fr/Publications/Memoires/Presentation.html) implique l'accord avec les conditions générales d'utilisation (http://www.numdam.org/conditions). Toute utilisation commerciale ou impression systématique est constitutive d'une infraction pénale. Toute copie ou impression de ce fichier doit contenir la présente mention de copyright.

\section{Numdam}


Colloque Anal. fonctionn.[1971, Bordeaux]

Bull. Soc. math. France,

Mémoire 31-32, 1972, p. 317-319.

SUR L'ECOULEMENT D'UN LIQUIDE VISQUEUX INCOMPRESSIBLE

DANS UN TUYAU AVEC PAROI PERMEABLE ET DEFORMABLI

par

Giovanni PROUSE

On va étudier le problème de l'écoulement d'un liquide visqueux incompressible dans un tuyau avec paroi perméable et déformable. Ce problème se pose, par exemple, dans l'étude de l'écoulement du sang dans les artères artificielles.

Pour éviter des complications formelles, nous allons simplifier le problème le plus possible, bien que les résultats gardent leur validité dans des conditions plus générales. On va, en tout cas, considérer le cas à deux dimensions d'espace, parce que les énoncés ne peuvent pas s'étendre directement aux problèmes en dimension 3 .

Soit $\Omega_{\varphi}$ I'ensemble du plan $x_{1}, x_{2}: \Omega_{\varphi}=\left\{\left\{x_{1}<\ell, \varphi\left(x_{1}, t\right)<x_{2}<k\right\}\right.$ avec frontière $\Gamma_{\varphi}$, formée par les lignes $\Gamma_{1}=\left\{x_{1}=0,0<x_{2}<k\right\}$. $\Gamma_{2}=x^{\prime}\left\{x_{1}=\ell, 0<x_{2}<k\right\}, \Gamma_{3, \varphi}^{\prime}={ }^{\prime}\left\{0<x_{1}<\ell, x_{2}=\varphi\left(x_{1}, t\right)\right\}$, $\Gamma_{3}^{\prime \prime}=\left\{0<x_{1}<l, x_{2}=k\right\}$, où $\varphi\left(x_{1}, t\right)$ est une fonction $\epsilon_{C}^{1}(R)\left(R=\left\{0, x_{1}: l, 0, t, T\right\}\right)$, avec $\varphi(0, t)=\varphi(l, t)=0$. $\Omega_{\varphi}$ représente le "tuyau" qu'on va considérer, correspondant à la section d'une couche infinie avec $x_{3}=0$. $\Gamma_{1}$ et $\Gamma_{2}$ représentent les sections de "entrée" et de"sortie" du tuyau, $\Gamma_{3, \varphi}^{\prime}$ et $\Gamma_{3}^{\prime \prime}$ les parois que I'on suppose perméables. On va aussi supposer que $\Gamma_{3}^{\prime \prime}$ est rigide, tandis que $\Gamma_{3, \varphi}^{\prime}$ est déformable; la déformation dépend de la pression exercée par le liquide sur la paroi selon une loi qu'on va établir plus loin.

L'écoulement d'un liquide de densité 1 et de viscosité u est gouverné par les équations de Navier-Stokes

$$
\begin{aligned}
& \frac{\partial u_{j}}{\partial t}-u_{\Delta u_{j}}+\sum_{k=1}^{2} u_{k} \frac{\partial u_{j}}{\partial x_{k}}+\frac{\partial p}{\partial x_{j}}=f_{j} \quad(j=1,2) \\
& \sum_{k=1}^{2} \frac{\partial u_{k}}{\partial x_{k}}=0
\end{aligned}
$$

où $\vec{f}(x, t)\left(x=\left\{x_{1}, x_{2}\right\}\right)$ est la force appliquée, $\vec{u}(x, t)$ la vitesse et $p(x, t)$ la pression du liquide.

Notre but consiste à déterminer, sous des conditions appropriées, le mouvement du liqui.de (i.e. le vecteur $\vec{u}$ ) et la configuration du tuyau (i.e. la fonction $\left.\varphi\left(x_{1}, t\right)\right)$. 
Le problème est évidemment "bien posé" du point de vue physique si on donne les conditions suivantes :

a) La configuration du tuyau en absence de pression. On va, pour cela, admettre qu'aucune pression n'agit sur la paroi du tuyau de l'extérieur et que, si la pression à l'intérieur est nulle, le tuyau est représenté par le rectangle $\Omega_{0}=\left\{0<x_{1}<l, 0<x_{2}<k\right\}$.

b) La relation entre la pression exercée par le liquide sur la paroi du tuyau et la déformation de la paroi même ( $i$.e. entre $p\left(x_{1}, \varphi\left(x_{1}, t\right), t\right)$ et $\varphi\left(x_{1}, t\right)$ ), Cette relation est interprétée par une inéquation variationnelle de type hyperbolique qui représente une formulation généralisée du problème de la barre vibrante avec la condition que la courbure dans chaque point de la barre n'ait pas des variations trop rapides $\left(\left|\frac{\partial 3 \varphi}{\partial \operatorname{tax} x_{1}^{2}}\right| \mathrm{M}\right)$.

c) Les conditions initiales du système, représentées par la vitesse $\vec{u}(x, 0)=\vec{u}_{0}(x)$ et les fonctions $\varphi\left(x_{1}, 0\right), \frac{\partial \varphi\left(x_{1}, 0\right)}{\partial t}$.

d) Les conditions aux limites

$$
\begin{array}{ll}
\frac{1}{2}|\vec{u}(x, t)|^{2}+p(x, t)=\alpha_{i}(x, t) & \left(x \in \Gamma_{i}, i=1,2\right) \\
p(x, t)=\beta(x, t) \vec{u}(x, t) \times \vec{v}_{\varphi}|\vec{u}(x, t)| & \left(x \in \Gamma_{3, \varphi}^{\prime} \Gamma_{3}^{\prime \prime}=\Gamma_{3, \varphi}\right) \\
\left|\vec{u}(x, t) \times \vec{v}_{\varphi}\right|=|\vec{u}(x, t)| & \left(x \in \Gamma_{\varphi}\right)
\end{array}
$$

où $\vec{v}_{\varphi}$ désigne la normale extérieure à $\Gamma_{\varphi}$. Equation (2) définit la valeur de "1'énergie totale" sur les sections $\Gamma_{1}$ et $\Gamma_{2} ;(4)$ impose la condition que sur $\Gamma_{\varphi}$ la vitesse est dirigée comme la normale à $\Gamma_{\varphi}$; finalement, (3) interprète la condition (non linéaire) de perméabilité, $\beta>0$ étant le coefficient de perméabilité de la paroi.

On démontre que le problème ainsi posé admet une solution, dans un sens convenable.

Soit $\Omega_{\varphi}$ un ouvert du plan $x_{1}, x_{2}$ satisfaisant la propriété du cône et soit $\eta(\Omega)$ la variété des vecteurs $\vec{v}(x)$ indéfiniment différentiables dans $\Omega$, avec divergence nulle et tels que $|\vec{v}(x) \times \vec{v}|=|\vec{v}(x)|$ ( $x \in \partial \Omega, \vec{v}$ normale extérieure). On désigne par $\mathrm{N}^{\sigma}(\Omega)(\sigma \geqslant 0)$ la fermeture de $\eta(\Omega)$ dans $\mathrm{H}^{\sigma}(\Omega)$ et par $\mathrm{D}(\mathrm{A})$ l'espace (hilbertien) des $\vec{u} \in N^{\prime}(\Omega)$ tels que $\vec{v} \rightarrow(\vec{u}, \vec{v})_{N^{l}(\Omega)}$ est continue dans la topologie de $\mathrm{N}^{\mathrm{O}}(\Omega), \mathrm{Vv}_{\mathrm{v}} \in \mathrm{N}^{\mathrm{I}}(\Omega)$. On pose, finalement, $\mathrm{V}_{\sigma}(\Omega)=\mathrm{D}\left(\mathrm{A}^{\sigma / 2}\right), \mathrm{V}_{\mathrm{o}}(\Omega)=\left(\mathrm{V}_{\mathrm{o}}(\Omega)\right)^{\prime}$, $\mathrm{v}_{-\sigma}(\Omega)=\left(\mathrm{v}_{\sigma}(\Omega)\right)^{\prime} \cdot$ 
Supposant que $\vec{f}(t)=\left\{f(x, t) ; x \in \Omega_{\varphi}\right\} \in L^{2}\left(O, T ; V_{\sigma-1}\left(\Omega_{\varphi}\right)\right)$, $\alpha_{i_{\infty}}(t)=\left\{\alpha_{i}(x, t) ; x \in \Gamma_{i}\right\} \in L^{2}\left(O, T ; L^{2}(\Gamma),\right), \beta(t)=\left\{\beta(x, t) ; x \in \Gamma_{3}, \varphi\right\}$ $\in L^{\infty}\left(0, T ; L^{\frac{1}{\infty}}\left(\Gamma_{3}, \varphi\right)\right), \varphi\left(x_{1}, t\right) \in C^{1}(R)$

et posant :

$$
\mathrm{b}_{\varphi}(\vec{u}(t), \vec{v}(t), \vec{w}(t))=\int_{\Omega_{\varphi}} \sum_{j_{l} k=1}^{2} u_{j}(x, t) \frac{\partial v_{k}(x, t)}{\partial x_{j}} w_{k}(x, t) d \Omega_{\varphi},
$$

on dira que $\vec{u}(t)=\left\{\vec{u}(x, t) ; x \in \Omega_{\varphi}\right\}$ est une solution en [0,T] de (I) satisfaisant les conditions aux limites (2), (3), (4') si

(i) $\vec{u}(t) \in L^{2}\left(0, T ; V_{\sigma+1}\left(\Omega_{\varphi}\right)\right) \cap L^{\infty}\left(0, T ; V_{\sigma}\left(\Omega_{\varphi}\right)\right) \cap H^{I}\left(O, T ; V_{\sigma-I}(\Omega \varphi)\right)$;

(ii) $\vec{u}(t)$ est une solution de I'équation

$\int_{0}^{T}\left\{\left\langle\vec{u}^{\prime}(t), \vec{h}(t)\right\rangle+u<A \vec{u}(t), \vec{h}(t)>+b_{\varphi}(\vec{u}(t), \vec{u}(t), \vec{h}(t))-\langle\vec{f}(t), \vec{h}(t)>\} d t=\right.$
$\int_{0}^{T}\left\{\sum_{i=1}^{2} \int_{\Gamma_{i}}\left(\alpha_{i}(x, t)-u_{l}^{2}(x, t)\right) \vec{h}(x, t) \times \vec{v}_{\varphi} d \Gamma_{i}+\int_{\Gamma_{3}^{\prime}, \varphi \Gamma_{3}^{\prime \prime} \beta(x, t) \vec{u}(x, t) \times \vec{v}_{\varphi}|\vec{u}(x, t)|}^{\left.\vec{h}(x, t) \times \vec{v}_{\varphi} d \Gamma_{3}\right\} d t}\right.$

$\forall h(t) \in L^{2}\left(0, T ; V_{l-\sigma}\left(\Omega_{\varphi}\right)\right)$, où $<,>$ désigne la dualité, $\forall t \in[0, T]$, entre $\mathrm{v}_{\sigma-1}\left(\Omega_{\varphi}\right)$ et $\mathrm{v}_{1-\sigma}\left(\Omega_{\varphi}\right)$.

Equation (5) est obtenue en multipliant la première de ( 1 ) par $h_{j}(x, t)$, intégrant sur $\Omega_{\varphi}[0, T]$ et en tenant compte de la deuxième équation (1) et de (2), (3), (4). On peut démontrer le théorème suivant :

THEOREME. - $\underline{\mathrm{Si}}\|\overrightarrow{\mathrm{f}}(\mathrm{t})\|_{L^{2}\left(0, T ; V_{\sigma-1}\left(\Omega_{\varphi}\right)\right)} \leqslant M_{1},\|B(t)\|_{L^{\infty}\left(0, T ; L^{\infty}\left(\Gamma_{3, \varphi}\right)\right.} \leqslant M_{2}$ $\forall \varphi \in C^{I}(R)$, avec $\varphi(0, t)=\varphi(l, t)=0,\left\|\alpha_{i}(t)\right\|_{L^{2}\left(O, T ; L^{2}\left(\Gamma_{i}\right)\right)} \leqslant M_{3}$, $\vec{u}_{0} \in v_{\sigma}\left(\Omega_{0}\right), \frac{1}{4}<\sigma<\frac{l}{2}$, alors le problème $\left.\left.\left.a\right), b\right), c\right)$, d) admet, dans le sens précisé, une solution, pour $T$ assez petit.

La démonstration de ce résultat et de quelques théorèmes auxiliaires se trouve dans les notes:

- "On the solution of a non-linear mixed problem for the Navier-Stokes equations in a time dependent domain, notes I, II, III ",

et :

- "On the motion of a viscous incompressible fluid in a tube with permeable and deformable wall",

à parâ̂tre aux Rend. Acc. Naz. Lincei, 1971. 